\title{
Comparison of different cytological tools with conventional diagnostic methods in diagnosis of Helicobacter pylori infection
}

\author{
Adlekha S.K ${ }^{1}$, Chadha $\mathbf{T}^{2}$ \\ ${ }^{1}$ Dr. Shashikant Adlekha, Associate Professor, Department of Pathology, ${ }^{2}$ Dr Tandra Chadha, Associate Professor, \\ Department of Microbiology; Maharaja Institute of Medical Sciences, Vizianagram, Andhra Pradesh, India.
}

Address for Correspondence: Dr. Shashikant Adlekha, Email: ruc.isha@gmail.com

\begin{abstract}
Background: Helicobacter Pylori (H pylori) infection causes severe gastrointestinal morbidity and mortality worldwide. Objective: To evaluate the usefulness of gastric crush cytology and imprint cytology with conventional histopathology and rapid urease tests. Materials and Methods: Antral biopsies were collected from 130 patients and evaluated for $H$ Pylori infection by imprint cytology, crush cytology and histopathological examination by different stains and rapid urease test. Results: 118 patients showed H. pylori infection by two or more methods. Giemsa histology, showed highest sensitivity, specificity, Positive predictive value (PPV), negative predictive value (NPV) and Youden's index (YI). Among cytology methods, crush cytology was found more effective in detecting $H$ pylori infection. Conclusion: $H$. pylori infection is associated with gastric mucosa changes like chronic active gastritis, atrophy, intestinal metaplasia, ulceration and carcinoma. Imprint cytology has high sensitivity and comparable predictive values to conventional diagnostic tools-histopathological examination and rapid urease test in detection of $H$. pylori infection.
\end{abstract}

Keywords: H pylori, Histopathology, Crush Cytology, Imprint Cytology

\section{Introduction}

$\mathrm{H}$ pylori infection is recognised one of the most important causative factor of gastroduodenal diseases. The prevalence of $\mathrm{H}$ Pylori infection shows marked geographical variation with maximum prevalence in developing countries [1]. The annual incidence of $\mathrm{H}$ Pylori infection is 0.3 to $0.7 \%$ in developed countries and $6-14 \%$ in developing countries [1].Various invasive and non invasive tests are employed for detection of $H$ Pylori infection. Non invasive tests include urea breath tests, serological test- IgG, IgM detection, salivary and urinary antibodies test and stool antigen test [3]. The invasive tests are endoscopy based tests, which include histipathological examination, cytological examinationCrush and imprint cytology, rapid urease test (RUT) and polymerase chain reaction. Cytological examination, such as imprint and crush smears have been used in detection of malignancy with sensitivity up to $95.2 \%$ [4]. These techniques are routinely not used in detection of $\mathrm{H}$ pylori infection. So we evaluated the usefulness of imprint and crush smears with conventional histopathological examination and RUT.

Manuscript received: $10^{\text {th }}$ November 2016

Reviewed: $24^{\text {th }}$ November 2016

Author Corrected: $4^{\text {th }}$ December 2016

Accepted for Publication: 14 ${ }^{\text {th }}$ December 2016

\section{Material and Methods}

The patients were selected on the basis of chief complaints of dyspepsia and the age of patients ranged from 14 to 86 years. Certain exclusion criteria were applied such as patients on proton pump inhibitor (PPI) therapy or any antibiotic therapy within last one month. Endoscopy was carried out using "Pentax" forward viewing oesophago gastro duodenoscope. The patients were taken for upper G.I. Endoscopy after making them fast overnight. The endoscopy was considered normal on visualizing mucosa which is pink in colour, smooth and lustrous 130 patients undergoing upper gastrointestinal endoscopy in the hospital-Sree Narayana Institute of Medical Sciences, Ernakulam, Kerala, India were enrolled in this prospective type of study. Three different diagnostic methods were usedhistology, cytology (Imprint and crush) and RUT. Three antral biopsy fragments were obtained from each patient and two samples sent for pathological examination in unfixed state and one sample being sent for RUT. Imprint smears were prepared from one fragment by keeping one fragment on a glass slide and gently touching it without pressing. Imprint slides were 


\section{Research Article}

air dried and stained for Giemsa stain, alcohol fixed and stained for $\mathrm{H} \& \mathrm{E}$. The imprinted tissue piece was crushed between two slides and slides stained with $\mathrm{H} \&$ $\mathrm{E}$ and Giemsa stains and second biopsy specimens were fixed in $10 \%$ formalin and processed for three micrometre thick sections and stained with $\mathrm{H} \& \mathrm{E}$ and Giemsa stains. H. pylori classically appear as small curved or s-shaped structures. Occasional coccoid forms may be seen and are difficult to be interpreted by routinely used stains. Immunohistochemical staining may be employed for detection of these forms.

On another biopsy fragment, RUT was performed by following method - Urea (2 g) was dissolved in $20 \mathrm{ml}$ double distilled water. 20 drops of phenol red was added to the solution and $\mathrm{pH}$ was adjusted between 6.8 and 6.9 by adding a drop of $\mathrm{N} / 10 \mathrm{HCl}$, if $\mathrm{pH}$ was greater or $\mathrm{N} / 10 \mathrm{Na} \mathrm{NaOH}$, if $\mathrm{pH}$ was less. Solution was having faint yellow tint at this stage.

This was transferred to sterile vial each containing $2 \mathrm{ml}$ in each vial. Biopsy material was added and the temperature was kept constant at $35-37^{\circ} \mathrm{C}$. Test was considered positive, if colour changed within 30 minutes and weekly positive, if the change occurred after 2 hours.
Histopathological changes of gastric mucosa were also assessed. Lymphoplasmacytic infiltrates without neutrophilic infiltration was regarded as chronic gastritis and with neutrophilic infiltration as chronic active gastritis. Atrophy of glands was regarded as atrophic gastritis and goblet cell metaplasia of glandular lining was regarded as intestinal metaplasia. Density of H. pylori was assessed according to visual analogue scale of updated Sydney grading system [5]. To increase the accuracy and prevent bias, positivity for two or more methods (by any of the stains, RUT) was considered as true positive. Sensitivity, specificity, PPV and NPV of different methods were computed and compared.

Sensitivity $=$ True positive $/$ True positive + False negative); Specificity $=$ True negative/ $($ True negative + False positive); PPV $=$ True positive/ $($ True positive + False positive) NPV = True negative/ (True negative + False negative).

Youden's index $=$ Sensitivity + Specificity -100 . Informed consent was taken from each patient and the study was approved by scientific research committee of the institution.

\section{Results}

130 persons with dyspeptic symptoms were enrolled in the present study with 68 males and 62 females with mean age of $49 \pm 9.7$ years. Taking the criteria of two or more positive results to be positive, 118 out of 130 patients tested positive for $H$ pylori infection. 94 patients tested positive for all the methods (Table 2). 110 patients tested positive by histological methods (108 by H \& E and 110 by Giemsa). RUT results showed 102 patients to be positive for $H$. pylori infection. Crush cytology examination showed positivity for H pylori infection in 104 and 102 patients by Giemsa and H\&E stains respectively. Imprint cytology examination showed positivity for $H$. pylori infection in 103 and 94 patients by Giemsaand H\&E stains respectively. Histopathological assessment (Table 1) of 130 patients showed chronic active gastritis in 76 patients, chronic gastritis in 20 patients, chronic active gastritis with intestinal metaplasia in 12 patients, chronic follicular gastritis in 10 patients and ulcerative changes in 6 patients. Dysplastic changes were not seen in any patient. Normal mucosal study was seen in 6 patients.

Table-1: Histopathological assessment of gastric mucosa.

\begin{tabular}{|c|c|c|c|}
\hline Histological diagnosis & No. of cases & HP Pos. & HP Neg. \\
\hline CAG & 76 & 70 & 06 \\
\hline CG & 20 & 16 & 04 \\
\hline CAGIM & 12 & 08 & 00 \\
\hline CFG & 10 & 10 & 00 \\
\hline Ulcer & 06 & 06 & 06 \\
\hline Normal & 06 & 00 & $\mathbf{N = 2 0}$ \\
\hline
\end{tabular}

CG-Chronic gastritis, CAG-Chronic active gastritis, CAGIM-Chronic active gastritis with intestinal metaplasia, CFGChronic follicular gastritis, HP-Helicobacter pylori. 
Research Article

Table-2: Categorisation of cases based on results of diagnostic tests.

\begin{tabular}{|c|c|c|c|c|c|c|c|c|}
\hline Cases & H \& E, B & G, B & G,C & H \& E, & G,I & H \& E, I & RUT & Final \\
\hline 94 & $\mathrm{P}$ & $\mathrm{P}$ & $\mathrm{P}$ & $\mathrm{P}$ & $\mathrm{P}$ & $\mathrm{P}$ & $\mathrm{P}$ & $\mathrm{P}$ \\
\hline 10 & $\mathrm{P}$ & $\mathrm{P}$ & $\mathrm{N}$ & $\mathrm{N}$ & $\mathrm{N}$ & $\mathrm{N}$ & $\mathrm{N}$ & $\mathrm{P}$ \\
\hline 4 & $\mathrm{P}$ & $\mathrm{P}$ & $\mathrm{P}$ & $\mathrm{P}$ & $\mathrm{N}$ & $\mathrm{N}$ & $\mathrm{N}$ & $\mathrm{P}$ \\
\hline 4 & $\mathrm{~N}$ & $\mathrm{~N}$ & $\mathrm{P}$ & $\mathrm{P}$ & $\mathrm{P}$ & $\mathrm{N}$ & $\mathrm{P}$ & $\mathrm{P}$ \\
\hline 4 & $\mathrm{~N}$ & $\mathrm{~N}$ & $\mathrm{~N}$ & $\mathrm{~N}$ & $\mathrm{P}$ & $\mathrm{N}$ & $\mathrm{P}$ & $\mathrm{P}$ \\
\hline 11 & $\mathrm{~N}$ & $\mathrm{~N}$ & $\mathrm{~N}$ & $\mathrm{~N}$ & $\mathrm{~N}$ & $\mathrm{~N}$ & $\mathrm{~N}$ & $\mathrm{~N}$ \\
\hline 1 & $\mathrm{~N}$ & $\mathrm{~N}$ & $\mathrm{~N}$ & $\mathrm{~N}$ & $\mathrm{P}$ & $\mathrm{N}$ & $\mathrm{N}$ & $\mathrm{N}$ \\
\hline 2 & $\mathrm{~N}$ & $\mathrm{P}$ & $\mathrm{P}$ & $\mathrm{N}$ & $\mathrm{N}$ & $\mathrm{N}$ & $\mathrm{N}$ & $\mathrm{P}$ \\
\hline
\end{tabular}

H \& E- Hematoxylin and Eosin, RUT- Rapid urease test, I-Imprint, C-Crush, B-Biopsy, N-Negative, P-Positive, No.Number

Table-3: Predictive values of different diagnostic tests.

\begin{tabular}{|c|c|c|c|c|c|}
\hline Diagnostic methods & Sensitivity & Specificity & PPV & NPV & YI \\
\hline H\&E, Histology & 92.18 & 100 & 100 & 54.54 & 92 \\
\hline Giemsa, Histology & 93.65 & 100 & 100 & 60 & 94 \\
\hline H\& E, Crush & 88.05 & 100 & 100 & 42.85 & 88 \\
\hline Giemsa, Crush & 89.39 & 100 & 100 & 46.15 & 89 \\
\hline Giemsa, Imprint & 88.05 & 92.30 & 99.15 & 42.85 & 80 \\
\hline H\&E, Imprint & 83.09 & 100 & 100 & 33.33 & 83 \\
\hline RUT & 88.05 & 100 & 100 & 42.85 & 88 \\
\hline
\end{tabular}

H \& E- Hematoxylin and Eosin, RUT-Rapid urease test, PPV-Positive predictive value, NPV-Negative predictive value, YI-Youden's index

Predictive values of different methods when computed and compared (Table 3), showed highest sensitivity of $93.65 \%$ for Giemsa histology followed by, in descending order- H \& E histology (92.18\%), Giemsa crush (89.39\%), Giemsa imprint (88.05\%), H \& E Crush (88.05\%), RUT (88.05\%) and H \& E Imprint (83.09\%). Specificity of all the methods was $100 \%$, except for Giemsa imprint, with specificity of $92.30 \%$. Similarly, Giemsa imprint showed PPV of $99.15 \%$ and all other methods showed PPV of 100\%. NPV was highest for Giemsa histology (60\%), followed in descending order by, $54.54 \%$ for H \& E histology, Giemsa crush (46.15\%), Giemsa imprint (42.85\%), H \& E crush (42.85\%), RUT (42.85\%) and H\&E imprint (33.3\%). YI when calculated was highest for Giemsa histology -94, followed by in descending order- H\&E histology- 92, Giemsa crush- 89, RUT- 92, H \& E crush-88, RUT-88, H \& E imprint- 83 and Giemsa imprint-80.

\section{Discussion}

H. pylori infection is associated with varying degree of inflammation and architectural distortion in different individuals. This variability is not only accounted by the variation in bacterial load/density, but also relies on immunogenicity of host/patient. In $2 \%$ of cases, $H$. pylori infection leads only to mild chronic gastritis or almost unremarkable mucosal change $[5,6]$. H. pylori infection diagnosis entails different methods each with different advantages and limitation. The most popular and widely used method is histopathological examination of antral biopsies, employing $\mathrm{H} \& \mathrm{E}$ stain [7]. Histopathological examination is not only very sensitive and specific method of diagnosing $H$. pylori infection, but also provides valuable information regarding the mucosal architectural distortion and atypia if any. The major limitation is that it is time consuming and expensive. Among cytology methods, crush cytology and imprint cytology are equally sensitive methods of diagnosing $H$. pylori infection. Imprint cytology, crush cytology and RUT are faster and results are available when patient is still in endoscopy unit. This results in early initiation of anti- $H$. pylori treatment. Imprint cytology and crush cytology by various rapid and routine stains leads to much earlier detection, in comparison to 2 hour time of RUT. In the present study, we evaluated the predictive values of 


\section{Research Article}

different methods. Giemsa biopsy has highest sensitivity, specificity, PPV, NPV and YI. Comparable sensitivity, specificity, PPV and NPV were seen for histopathology, Crush and imprint cytology. Various studies have compared the predictive values of different stains in diagnosing $H$. pylori infection. Misra et al [8] reported equal sensitivity and specificity of imprint cytology as that of biopsy examination. When evaluated in terms of YI, highest YI was found for Giemsa histology. YI validates a technique by taking both sensitivity and specificity into account. Low NPV noted in this study for different methods can be attributed to false negative cases reported. Low bacterial load and multifocality of the bacteria can lead to false negative cases in cytological smears, as sparse $H$. pylori are difficult to interpret amidst the dirty background of smears. While imprint cytology represents the superficial part of the biopsy, crush cytology represents entire biopsy specimen and has comparable sensitivity and specificity to histopathology. Even in histopathology examination, specimen processing can lead to false negative result due to partial loss of area in or beneath the surface mucosal layer, especially in set up of low bacterial density $[8,9]$. This is in concordance with the present study findings, as all false negative cases seen in different methods, had low $H$. pylori density.

\section{Conclusion}

Cytological tools-Imprint cytology and crush cytology are rapid inexpensive methods of diagnosing $H$. pylori infection. It has comparable predictive values to histopathological examination and RUT. Cytological smear examination and biopsy should be used in conjunction, as rapid diagnosis and architectural assessment of gastric mucosa is essential for effective management of the patient.

Funding: Nil, Conflict of interest: None initiated, Permission from IRB: Yes

\section{References}

1. Malatey HM, Hoda N. Helicobacter pylori infection: genetic and environmental influences. Best practice \& Research ClinGasteroenterol. 2007; 21(2):205-14.
2. Kaore NM, Nagdeo NV, Thombare VR. Comparative evaluation of the diagnostic tests for Helicobacter pylori and dietary influence for its acquisition in dyspeptic patients: a rural hospital based study in central India. JCDR 2012;6(4):636-41.

3. Faraker CA. Diagnosis of Helicobacter pylori in gastric brush and biopsy specimens stained by Romanowsky and immunocytochemical methods: Comparison with the CLO test. Cytopathology 1996; 7:108-19.

4. Kochhar R, Bhasin DK, Rajwanshi A, Gupta SK, Malik AK, Mehta SK. Crush preparations of gastroesophageal biopsy specimens in the diagnosis of malignancy. Acta Cytol. 1990;34(2):214-6.

5. Bayerdörffer E, Oertel H, Lehn N, Kasper G, Mannes GA, Sauerbruch T, Stolte M. Topographic association between active gastritis and Campylobacter pylori colonisation. J ClinPathol.1989;42(8):834-39.

6. Prasad S, Mathan M, Chandy G, Rajan DP, Venkateswaran S, Ramakrishna BS, Mathan VI.: Prevalence of Helicobacter pylori in southern Indian controls and patients with gastroduodenal disease. J GastroenterolHepatol 1994;9(5):501-6.

7.T revisani L, Sartori S, Ruina M, Caselli M, Abbasciano V, Grandi E, Forini E. Touch cytology. A reliable and cost-effective method for diagnosis of Helicobacter pylori infection. Dig Dis Sci. 1997;42 (11):2299-303.

8. Misra SP, Dwivedi M, Misra V, Gupta SC. Imprint cytology - a cheap, rapid and effective method for diagnosing Helicobacter pylori. Postgrad Med J 1993; 69(810):291-5.

9. Debongnie JC, Donnay M, Mairesse J. Gastrospirillum hominis ("Helicobacter heilmanii"): a cause of gastritis, sometimes transient, better diagnosed by touch cytology? Am J Gastroenterol. 1995; 90 (3):411-6.

\section{How to cite this article?}

Adlekha S.K, Chadha T. Comparison of different cytological tools with conventional diagnostic methods in diagnosis of Helicobacter pylori infection.Trop J Path Micro 2016;2(3):164-167.doi: 10.17511/jopm.2016.i03.14 